\title{
Synthesis of polyaniline/ $\mathrm{ZrO}_{2}$ nanocomposites and their performance in $\mathrm{AC}$ conductivity and electrochemical supercapacitance
}

\author{
B P PRASANNA ${ }^{1,2}$, D N AVADHANI $^{3}$, H B MURALIDHARA ${ }^{4}$, K CHAITRA $^{4}$, \\ VINNY ROSE THOMAS ${ }^{4}$, M REVANASIDDAPPA ${ }^{5}$ and N KATHYAYINI ${ }^{4, *}$ \\ ${ }^{1}$ C.M.R.T.U. Research Centre, Tumkur University, Tumkur 572 101, India \\ ${ }^{2}$ Department of Physics, School of Engineering and Technology, Jain University, Bangalore 562 112, India \\ ${ }^{3}$ Department of Physics, R.V. College of Engineering, Bangalore 560 059, India \\ ${ }^{4}$ Centre for Emerging Technologies, Jain University, Bangalore 562 112, India \\ ${ }^{5}$ Department of Chemistry, PESIT Bangalore South Campus, Bangalore 560 100, India
}

MS received 8 June 2015; accepted 3 December 2015

\begin{abstract}
Polyaniline/zirconium oxide $\left(\mathrm{PANI} / \mathrm{ZrO}_{2}\right)$ nanocomposites have been synthesized by incorporating $\mathrm{ZrO}_{2}$ nanoparticles into the PANI matrix via liquid-liquid interfacial polymerization method. The composite formation and structural changes in $\mathrm{PANI} / \mathrm{ZrO}_{2}$ nanocomposites were investigated by powder $\mathrm{X}$-ray diffraction (PXRD), scanning electron microscopy (SEM) and Fourier transform infrared spectroscopy (FT-IR). PXRD pattern of PANI/ZrO 2 nanocomposites exhibited sharp and well-defined peaks of monoclinic phase of $\mathrm{ZrO}_{2}$ in PANI matrix. SEM images of the composites showed that $\mathrm{ZrO}_{2}$ nanoparticles were dispersed in the PANI matrix. The FT-IR analysis revealed that there was strong interaction between PANI and $\mathrm{ZrO}_{2}$. AC conductivity and dielectric properties of the nanocomposites were studied in the frequency range, $50-10^{6} \mathrm{~Hz}$. AC conductivity of the nanocomposites obeyed the power law indicating the universal behaviour of disordered media. The nanocomposites showed high dielectric constant in the order of $10^{4}$, which could be related to dielectric relaxation phenomenon. Further, the materials were checked for their supercapacitance performance by using cyclic voltammetry (CV), chronopotentiometry (CP) and electrochemical impedance spectroscopy (EIS). Among the synthesized nanocomposites, $\mathrm{PANI} / \mathrm{ZrO}_{2}-25$ wt. \% showed a higher specific capacitance of $341 \mathrm{~F} \mathrm{~g}^{-1}$ at $2 \mathrm{~m} \mathrm{Vs}^{-1}$ and good cyclic stability with capacitance retention of about $88 \%$ even after 500 charge-discharge cycles.
\end{abstract}

Keywords. Polyaniline; $\mathrm{ZrO}_{2}$ nanoparticles; interfacial polymerization; dielectric constant; pseudocapacitors.

\section{Introduction}

Polyaniline (PANI) is one of the promising conducting polymers due to its easy and cost-effective synthesis, stability in air and good electrical conductivity. PANI and their composites materials finds large variety of applications in lightemitting diodes [1], anti-corrosion coatings [2], gas sensors [3,4], memory devices catalysis [5], solar cells [6] and electrochemical energy storage $[7,8]$. Nanostructured conducting polyaniline with high surface area and porosity gives good performance in energy storage devices because of their distinctive characteristics of conducting pathways, surface interactions and nanoscale dimensions [9-11]. The morphology of conducting polymers also plays an important role in their electrochemical performances [12]. Different methods are being employed to synthesize polyaniline, such as template method [13], seeding method [14], interfacial polymerization [15] rapid mixing reaction [16], dilute polymerization [17] and electrochemical method [18]. Among these synthesis methods, interfacial polymerization has achieved more

\footnotetext{
*Author for correspondence (nkathyayini45@gmail.com)
}

attention due to its easiness, environmentally benign nature, large-scale production-ability and also it has a capability to produce high-quality polyaniline nanostructures with control of their morphology and size [19-21]. To improve the electrical and electrochemical performances, lot of efforts have been taken to synthesize PANI with different nanostructures such as nanofibres, nanoparticles, nanotubes, etc. Literature reveals that nanoparticles such as Ag [22], Ni [23], $\mathrm{MnO}_{2}$ [24], $\mathrm{SnO}_{2}$ [25], $\mathrm{TiO}_{2}$ [26], etc. could act as conductive pillers between the PANI chains that would result in enhanced electrical conductivity of nanocomposites. The electrochemical activity of PANI in its pure form could be a serious problem due to the volumetric shrinkage during ejection of counter ions and also it has low reversibility and stability for supercapacitors. To solve these problems and explore potential synergistic effects, we propose $\mathrm{PANI} / \mathrm{ZrO}_{2}$ nanocomposites as electrode material for supercapacitors.

Zirconium dioxide $\left(\mathrm{ZrO}_{2}\right)$ is an important transition metal oxide and finds wide applications in the field of catalysts [27], coatings [28], fuel cells [29] and sensors [30]. Further, $\mathrm{ZrO}_{2}$ exhibit high melting point, thermal expansion coefficient and stability that enable its use in several other engineering applications. Unfortunately, there are a very few efforts 
on developing PANI/ZrO $\mathrm{Zr}_{2}$ nanocomposites till now. Gupta et al [31] have synthesized PANI/ZrO $\mathrm{Zr}_{2}$ hybrid nanocomposites by chemical oxidative method and studied their electrical transport and optical properties. Rajeev Jain et al [32] have developed a sensitive esomeprazole sensor based on PANI/ $\mathrm{ZrO}_{2}$ nanocomposites and found that sensor exhibited an excellent electrocatalytic activity. Asif Ali Khan et al [33] have synthesized advanced nanocomposite-polyaniline zirconium titanium phosphate cation-exchanger and the material exhibited good performance for ion-exchange process. Tarique Anwer et al [34] have prepared $\mathrm{PANI} / \mathrm{ZrO}_{2}$ nanocomposites by in situ oxidative polymerization method and the material exhibited greater electrical conductivity and poorer isothermal and cyclic stability than pure PANI. However, pure $\mathrm{ZrO}_{2}$ has properties like high density, hardness, biocompatibility and mechanical strength that make the material suitable for its wide applications. But the application of pure $\mathrm{ZrO}_{2}$ in energy storage devices is not much explored due to its poor electrochemical performance. In recent literature survey, there are a few illustrations of $\mathrm{ZrO}_{2}$ and its composites being studied for their electrochemical supercapacitance properties. For instance, Mahdi Nasibi et al [35] have reported specific capacitance of $43.2 \mathrm{~F} \mathrm{~g}^{-1}$ for $\mathrm{ZrO}_{2} /$ carbon black as a possible electrode for electrochemical capacitor in $2 \mathrm{M}$ $\mathrm{KCl}$ electrolyte at the scan rate of $10 \mathrm{~m} \mathrm{Vs}^{-1}$. Wei Zhang et al [36] have prepared ultrafine $\mathrm{ZrO}_{2}$ by a one-step sol-gel method and reported a specific capacitance of $95 \mathrm{~F} \mathrm{~g}^{-1}$ in $6 \mathrm{M} \mathrm{KOH}$ electrolyte at scan rate of $5 \mathrm{~m} \mathrm{Vs}^{-1}$. Ko et al [37] have prepared amorphous $\mathrm{V}_{2} \mathrm{O}_{5}$ with $7 \% \mathrm{ZrO}_{2}$ dopant by one-pot spray pyrolysis and reported capacity retention of $83 \%$ after 50 cycles. These studies were found interesting and we have doped different weights $\%$ of $\mathrm{ZrO}_{2}$ on PANI by liquid-liquid interfacial polymerization and checked supercapcitor performance by $\mathrm{CV}, \mathrm{CP}$ and $\mathrm{AC}$ impedance techniques. The introduction of $25 \mathrm{wt} \%$ of $\mathrm{ZrO}_{2}$ and its intercalation in the PANI matrix was found to influence significantly on the surface morphology and electrochemical properties of the prepared nanocomposites and which may be attributed to the reduced oxygen vacancies defect level band due to the increasing oxycations in various oxidation states. Therefore, investigating the electrochemical properties of $\mathrm{PANI} / \mathrm{ZrO}_{2}$ nanocomposites was more valuable for supercapacitor electrode applications compared to earlier reports.

\section{Experimental}

\subsection{Materials}

Aniline $\left(\mathrm{C}_{6} \mathrm{H}_{5} \mathrm{NH}_{2}\right)$, ammonium peroxydisulfate $\left.\left(\mathrm{NH}_{4}\right)_{2} \mathrm{~S}_{2} \mathrm{O}_{8}\right)$, hydrochloric acid $(\mathrm{HCl})$, chloroform $\left(\mathrm{CCl}_{4}\right)$, zirconium oxide nanoparticles $\left(\mathrm{ZrO}_{2}\right)$, used in this work were of analytical grade and purchased from s.d. Fine Chemicals, Mumbai, India. Aniline was doubly-distilled at its boiling point $184^{\circ} \mathrm{C}$ before use. Deionized water was used throughout the work.

\subsection{Preparation of $\mathrm{PANI} / \mathrm{ZrO} \mathrm{O}_{2}$ nanocomposites}

$\mathrm{PANI} / \mathrm{ZrO}_{2}$ nanocomposites were synthesized by oxidative interfacial polymerization of aniline in the presence of $\mathrm{ZrO}_{2}$ nanoparticles using ammonium persulphate (APS) as an oxidant in acidic medium. Aniline $(0.1 \mathrm{M})$ in $20 \mathrm{ml}$ chloroform (solution A) and APS $(0.1 \mathrm{M})$ in $20 \mathrm{ml} \mathrm{HCl}(1 \mathrm{M})$ (solution B) were dissolved separately and stirred for $1 \mathrm{~h} . \mathrm{ZrO}_{2}$ nanoparticles with different wt.\% (5-25\%) were dispersed in solution (B) and sonicated for $1 \mathrm{~h}$ to reduce aggregation of $\mathrm{ZrO}_{2}$ nanoparticles. Then solution (B) was carefully transferred into solution (A), generating interface between two solutions. The reaction was allowed to proceed for $12 \mathrm{~h}$. At the end of the reaction, $\mathrm{PANI} / \mathrm{ZrO}_{2}$ composite formed was collected by filtration, washed with distilled water and acetone repeatedly until filtrate was colourless. The collected composite was dried at $80^{\circ} \mathrm{C}$ until constant weight was attained.

\subsection{Measuring methods}

Powder X-ray diffraction spectrum (PXRD) of PANI/ZrO $\mathrm{Zr}_{2}$ nanocomposites were recorded on a Bruker AXS D8 X-ray diffractometer using $\mathrm{CuK} \alpha$ radiation $(\lambda=1.5406 \AA)$ at a scanning rate of $2.0^{\circ} \mathrm{min}^{-1}$ using a voltage of $40 \mathrm{kV}$. Scanning electron microscope (SEM) images of nanocomposites were recorded using the Hitachi/SU-520 instrument. Fourier-transform infrared spectra (FT-IR) of the samples were recorded on Thermo Nicolet Avatar/370 spectrometer with $4 \mathrm{~cm}^{-1}$ resolution using $\mathrm{KBr}$ pellet technique.

Among the prepared nanocomposites of PANI/ZrO $\mathrm{Zr}_{2}$ with varied percentage of doped $\mathrm{ZrO}_{2}, \mathrm{PANI} / \mathrm{ZrO}_{2}-15$ wt. $\%$ and $\mathrm{PANI} / \mathrm{ZrO}_{2}-25$ wt.\% nanocomposites were selected to study their AC conductivity and electrochemical behaviour. AC conductivity response was measured for the samples by making $1 \mathrm{~cm}$ diameter pellets under a hydraulic pressure $10 \mathrm{Kpsi}$ and then applying conducting silver paste to form the electrodes in contact with circular faces. The samples were then held between two nominally springloaded copper plates and the AC parameters were measured at $300 \mathrm{~K}$ using Hioki (Japan) Model 3532-50 programmable LCR meter at the selected frequencies in the range from $50 \mathrm{~Hz}$ to $1 \mathrm{MHz}$. Electrochemical supercapacitance for these materials was performed on a CHI 660E electrochemical workstation. The working electrodes were prepared by coating a mixture of $85 \mathrm{wt} . \%$ of active material with 10 wt.\% Ketjen black conductive material and 5 wt.\% polyvinylidene fluoride (PVDF) binder dissolved in NMP as a solvent onto the nickel foils. The active masses of materials coated on nickel foils were found to be equal to $0.5 \mathrm{mg}$. Further, the electrodes were dried in a vacuum oven at $80^{\circ} \mathrm{C}$ overnight. $1 \mathrm{M} \mathrm{Na}_{2} \mathrm{SO}_{4}$ is used as electrolyte. Cyclic voltammetry (CV), chronopotentiometry (CP), electrochemical impedance spectroscopy (EIS) and cycle life test were carried out to assess the electrochemical properties of the devices. CV experiments were performed at varying scan rates. Galvanostatic charge discharge 
(GCD) was conducted with different current densities. EIS was performed on a fully discharged cell by sweeping frequencies from $100 \mathrm{kHz}$ to $0.01 \mathrm{~Hz}$ at amplitude of $5 \mathrm{mV}$. The cycling performance was charged and discharged for 500 cycles at a constant current density of $0.5 \mathrm{~A} \mathrm{~g}^{-1}$.

\section{Results and discussion}

\subsection{Characterization of materials}

$\mathrm{PANI} / \mathrm{ZrO}_{2}$ nanocomposites with varied wt. $\% \mathrm{ZrO}_{2}$ nanoparticles were characterized for their structural and morphological properties by PXRD, FTIR and SEM analytical techniques. Analysis of X-ray diffraction pattern of PANI/ $\mathrm{ZrO}_{2}-15 \%$ nanocomposites in figure 1a shows typical four diffraction peaks centered at 23.85, 27.98, 31.27 and $33.99^{\circ}$, representing Bragg reflections from $\left(\begin{array}{lll}1 & 1 & 0\end{array}\right),\left(\begin{array}{lll}1 & 1 & 1\end{array}\right),\left(\begin{array}{lll}1 & 1 & 1\end{array}\right)$ and (2 00 ) planes of $\mathrm{ZrO}_{2}$ (monoclinic phase) inductive of its crystalline nature (JCPDS card no. 37-1484) in polyaniline matrix. Average crystalline size was calculated by using Scherrer's formula, $D=0.9 \lambda / \beta \cos \theta$, where $\beta$ is the line broadening at the full-width at half maximum (FWHM) of the most intense peaks $\left(\begin{array}{lll}\overline{1} & 1 & 1\end{array}\right)$ and $\left(\begin{array}{lll}1 & 1 & 1\end{array}\right)$ and $\theta$ the Bragg angle and $\lambda$ the X-ray wavelength. The calculated average crystalline sizes were found to be 25.68 and $23.53 \mathrm{~nm}$, respectively.

Figure $1 \mathrm{~b}$ shows the FT-IR spectra of $\mathrm{PANI} / \mathrm{ZrO}_{2}$ nanocomposite. The vibration peaks at 1569.4, 1497.2 1444.9, $1295.1,1143.6$ and $820.3 \mathrm{~cm}^{-1}$ correspond to most of the characteristic peaks for PANI, as described in literature [38]. The strong absorption peaks corresponding to the stretching of quinonoid assigned at $1569.4 \mathrm{~cm}^{-1}$, benzenoid rings assigned at $1497.2 \mathrm{~cm}^{-1}, \mathrm{C}-\mathrm{N}$ stretching assigned at 1295.1 $\mathrm{cm}^{-1}, \mathrm{C}=\mathrm{N}$ stretching of a secondary aromatic amine assigned at $1143.6 \mathrm{~cm}^{-1}$ may be due to the presence of anion radical of acid molecules which is supposed to be absorbed to counterbalance the positive charge on the nitrogen atom of PANI during protonation and aromatic $\mathrm{C}-\mathrm{H}$ out-of-plane bending is assigned at $820.3 \mathrm{~cm}^{-1}$. The peaks at 743.2 and $508.4 \mathrm{~cm}^{-1}$, due to $\mathrm{Zr}-\mathrm{O}_{2}-\mathrm{Zr}$ asymmetric and $\mathrm{Zr}-\mathrm{O}$ stretching modes, respectively, confirm the formation of $\mathrm{ZrO}_{2}$ phases [39]. However, the small shifting in peaks are due to some sort of interaction between the PANI and $\mathrm{ZrO}_{2}$ nanoparticles in the composites [34].

Figure 1c shows the SEM images of $\mathrm{PANI} / \mathrm{ZrO}_{2}$ nanocomposite. It should be noted that nanosized $\mathrm{ZrO}_{2}$ particles were well dispersed in the matrix PANI and just a partial agglomeration in composites with the content of $\mathrm{ZrO}_{2}$ could be detected. Such a developed network structure contributes to enhanced conductivity of the nanocomposite material and is favourable to the diffusion of electrolyte solution and ion migration in the electrochemical process.

\subsection{AC conductivity studies}

Using the values of equivalent parallel capacitance, $C_{\mathrm{p}}$, impedance, $Z$ and phase angle, $\varphi$, recorded by the LCR
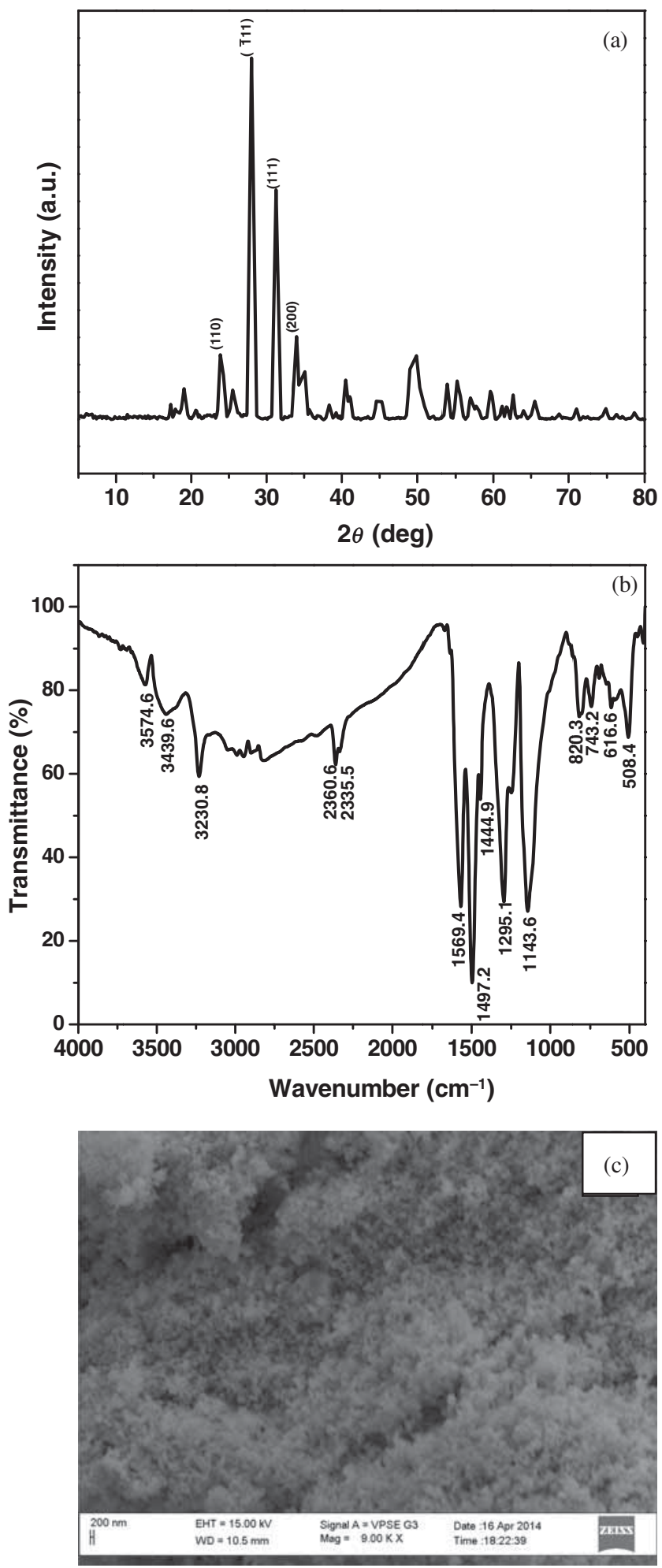

Figure 1. The (a) PXRD spectra, (b) FT-IR spectra and (c) SEM of $\mathrm{PANI} / \mathrm{ZrO}_{2}-15 \%$ nanocomposites.

meter at a selected frequency, $f$, dielectric and conductivity parameters are calculated using the following equations.

$$
\varepsilon^{\prime}=\frac{d C_{\mathrm{p}}}{A \varepsilon_{0}},
$$




$$
\begin{aligned}
& \varepsilon^{\prime \prime}=\varepsilon^{\prime} \tan \delta \text { or } \tan \delta=\frac{\varepsilon^{\prime}}{\varepsilon^{\prime \prime}}, \\
& \sigma^{\prime}(\omega)=\omega \varepsilon_{0} \varepsilon^{\prime \prime} \\
& \sigma^{\prime \prime}(\omega)=\omega \varepsilon_{0} \varepsilon^{\prime},
\end{aligned}
$$

where $C_{\mathrm{p}}$, the capacitance measured in pf; $A$ and $d$ the area and thickness of the samples, respectively; $\omega=2 \pi f$ and $\tan \delta$, dielectric loss with $\delta$ being phase angle. The $\varepsilon^{\prime}$ and $\varepsilon^{\prime \prime}$ are, respectively, the real and imaginary parts of the complex dielectric constant $\varepsilon(f)=\varepsilon^{\prime}(f)-i \varepsilon^{\prime \prime}(f)$. similarly, $\sigma^{\prime}$ and $\sigma^{\prime \prime}$ are, respectively, the real and imaginary parts of the complex AC conductivity, $\sigma(f)=\sigma^{\prime}(f)-i \sigma^{\prime \prime}(f)$.

Figure 2 shows AC conductivity response of PANI/ $\mathrm{ZrO}_{2}-15 \%$ and $\mathrm{PANI} / \mathrm{ZrO}_{2}-25 \%$ nanocomposites from $50 \mathrm{~Hz}$ to $1 \mathrm{MHz}$ frequency range. The $\mathrm{AC}$ response of both the composites exhibits a frequency independent conductivity in the low frequency region up to $10 \mathrm{kHz}$ and then increased as the frequency increased. This frequency independent plateau followed by a high frequency dispersed region in these composites obeyed the power law indicating the universal behaviour of the AC conductivity in disordered media [40]. Also in this dispersive region macroscopic conductivity was enhanced by the shorter length paths and the capacitive effects giving rise to polarization. The electrode polarization effects with the space charge polarization contributed to the increase in conductivity of the nanocomposites [41]. Table 1 shows the conductivity of different wt.\% of $\mathrm{ZrO}_{2}$ nanoparticles in PANI at selected frequency $500 \mathrm{kHz}$. Notice that conductivity

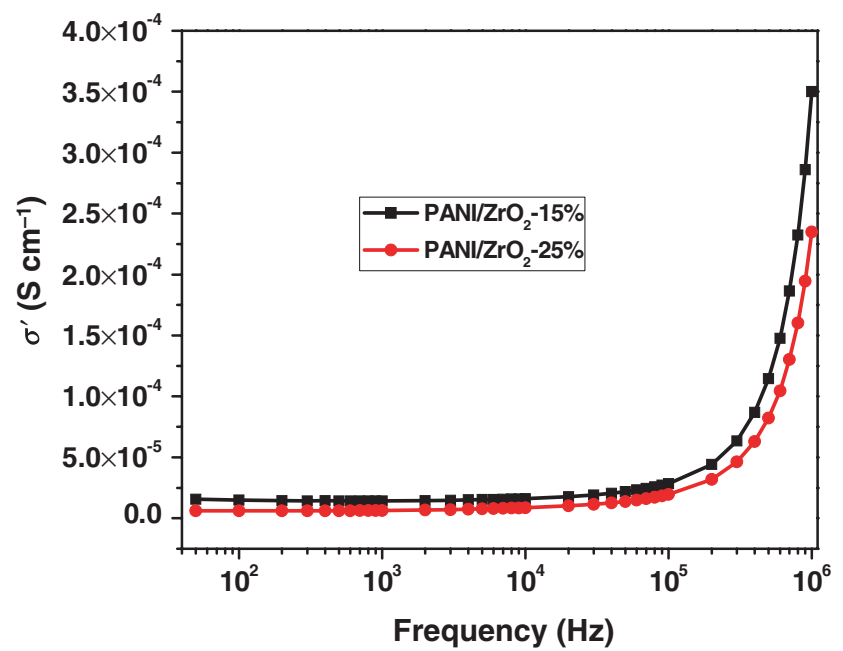

Figure 2. Frequency dependence of AC conductivity of PANI/ $\mathrm{ZrO}_{2}$ nanocomposites. decreases with increasing wt.\% of $\mathrm{ZrO}_{2}$ of the composite. The increased loading of $\mathrm{ZrO}_{2}$ nanoparticles might have increased the disorderliness in the composites and also $\mathrm{ZrO}_{2}$ nanoparticles could possibly induce conformational changes to PANI matrix, leading to a reduction in the delocalization length, which is reflected with a decrease in conductivity.

Figure 3 shows the variation of real part of dielectric constant $\left(\varepsilon^{\prime}\right)$ with frequency $(f)$ for $\mathrm{PANI} / \mathrm{ZrO}_{2}-15 \%$ and $\mathrm{PANI} / \mathrm{ZrO}_{2}-25 \%$ nanocomposites. The obtained high dielectric constant values of the order $10^{4} \mathrm{Scm}^{-1}$ are related to the effects of electrode polarization and space charge polarization [42]. The decrease of dielectric constant with respect to frequency is due to the fact that the nanocomposite acting as a semiconducting system with mobile polaron/bipolaron which is free to move along the chain, whereas the bound charges (dipoles) which have only restricted mobility resulting in strong polarization in the system. Thus, upon increase in the frequency of the applied field, the dipoles present in the system cannot reorient themselves quickly in response to the applied field reducing the dielectric constant [43]. Table 1 shows the dielectric constant of different wt. $\%$ of $\mathrm{ZrO}_{2}$ nanoparticles in PANI at selected frequency of $1 \mathrm{kHz}$. These results are in accordance with the observed conductivity behaviour of $\mathrm{PANI} / \mathrm{ZrO}_{2}$ nanocomposites. The nanocomposite with 15 wt. $\% \mathrm{ZrO}_{2}$ shows higher value of real dielectric constant due to dielectric relaxation process and the composite with 25 wt. $\% \mathrm{ZrO}_{2}$ shows slightly low dielectric constant because the relaxation mechanism is relatively slow compared to resonant electronic transitions or molecular vibrations.

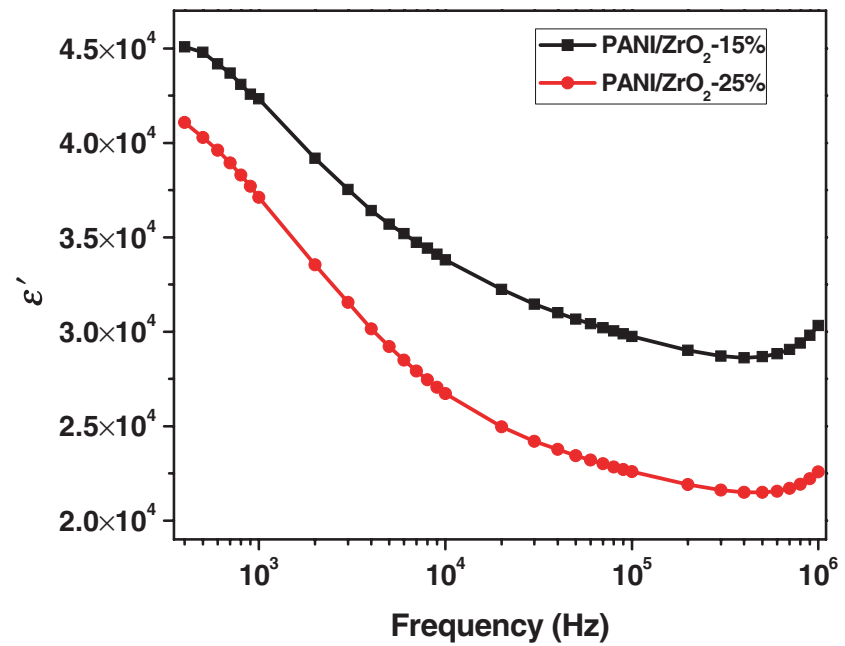

Figure 3. Frequency dependence of real dielectric constant of $\mathrm{PANI} / \mathrm{ZrO}_{2}$ nanocomposites.

Table 1. Summary of AC conductivity and dielectric response of $\mathrm{PANI} / \mathrm{ZrO}_{2}$ nanocomposites.

\begin{tabular}{lcccc}
\hline Composites & $\begin{array}{c}\text { Conductivity at } \\
500 \mathrm{kHz}, \sigma^{\prime}\left(\mathrm{S} \mathrm{cm}^{-1}\right)\end{array}$ & $\begin{array}{c}\text { Real dielectric constant } \\
\text { at } 1 \mathrm{kHz}, \varepsilon^{\prime}\end{array}$ & $\begin{array}{c}\text { Dielectric loss at } \\
1 \mathrm{kHz}, \tan \delta\end{array}$ & $\begin{array}{l}\text { Relaxation time, } \tau(\mathrm{s}) \\
\mathrm{PANI} / \mathrm{ZrO}_{2}-15 \%\end{array}$ \\
$\mathrm{PANI} / \mathrm{ZrO}_{2}-25 \%$ & $1.146 \times 10^{-4}$ & $4.234 \times 10^{4}$ & 3.557 & $2.653 \times 10^{-5}$ \\
\hline
\end{tabular}




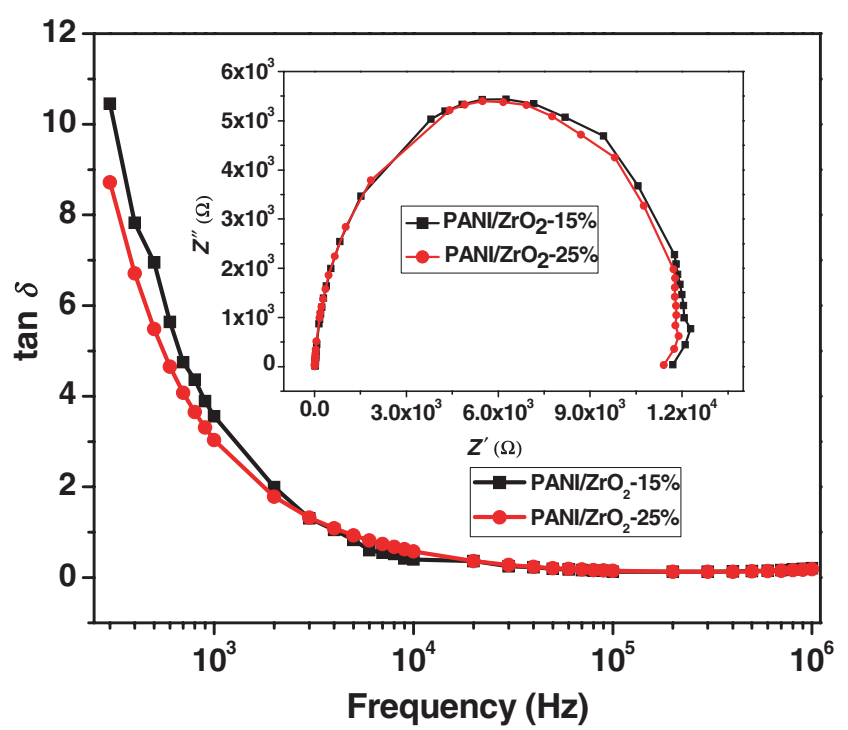

Figure 4. Frequency dependence of dissipation factor of $\mathrm{PANI} / \mathrm{ZrO}_{2}$ nanocomposites. Inset: complex plane impedance of $\mathrm{PANI} / \mathrm{ZrO}_{2}$ nanocomposites.

Figure 4 shows the variation of dissipation factor $(\tan \delta)$ with respect to frequency $(f)$ for $\mathrm{PANI} / \mathrm{ZrO}_{2}-15 \%$ and PANI/ $\mathrm{ZrO}_{2}-25 \%$ nanocomposites. The high loss tangent at low frequency in both the composites may be attributed to DC conduction losses. It is observed that the dielectric loss decreases as a function of frequency. The loss tangent for PANI/ZrO $\mathrm{Zr}_{2}$ $15 \%$ has a rather high value of 10.5 at $300 \mathrm{~Hz}$, which decreases with increasing frequency, reaching a value of 0.46 at $1 \mathrm{MHz}$. Table 1 shows the dissipation factor for different wt.\% of $\mathrm{ZrO}_{2}$ nanoparticles in PANI at selected frequency of $1 \mathrm{kHz}$. We see that dielectric loss also decreases with increase in wt.\% of $\mathrm{ZrO}_{2}$. This is expected because conductivity decreases with increase in wt.\% of $\mathrm{ZrO}_{2}$. This revealed the interaction of highly polar and semiconducting PANI with the stable semiconducting $\mathrm{ZrO}_{2}$ dispersant. The plot inset of figure 4 shows the complex plane impedance plots for $\mathrm{PANI} / \mathrm{ZrO}_{2}-15 \%$ and $\mathrm{PANI} / \mathrm{ZrO}_{2}-25 \%$ nanocomposites. For both composites including almost semicircular shape of the plot indicates the absence of contact effects and that relaxation nearly follows the Debye model with a single relaxation. The corresponding relaxation time, $\tau$, was obtained from this plot using the relation

$$
\tau=\frac{1}{2 \pi f_{\mathrm{p}}},
$$

where $f_{\mathrm{p}}$ is the frequency corresponding to peak position of the plot.

The relaxation time for different wt. $\%$ of $\mathrm{ZrO}_{2}$ nanoparticles in PANI is shown in table 1 . We see that relaxation time increases with increasing wt. $\%$ of $\mathrm{ZrO}_{2}$ of the composite. Since relaxation is related to charge transport by hopping mechanism [44], an increase in relaxation time indicates the increase in hopping length due to stretching effect of the
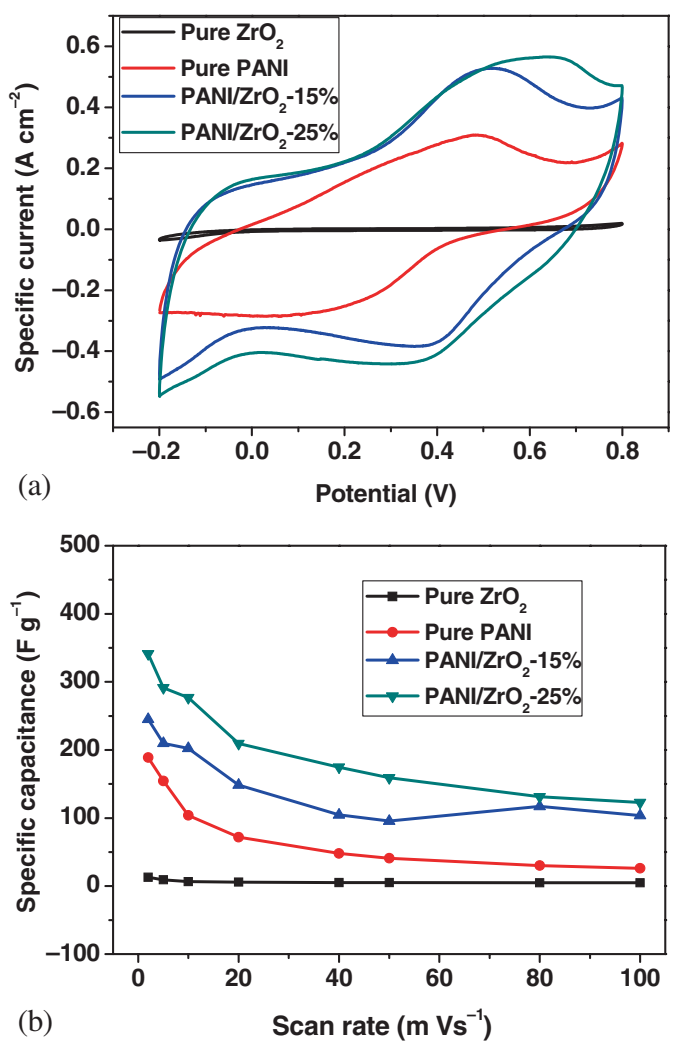

Figure 5. (a) $\mathrm{CV}$ curves of pure $\mathrm{ZrO}_{2}, \mathrm{PANI}$ and $\mathrm{PANI} / \mathrm{ZrO}_{2}$ nanocomposites at a scan rate of $5 \mathrm{~m} \mathrm{Vs}^{-1}$, (b) specific capacitance as a function of scan rate of pure $\mathrm{ZrO}_{2}, \mathrm{PANI}$ and $\mathrm{PANI} / \mathrm{ZrO}_{2}$ nanocomposites.

$\mathrm{ZrO}_{2}$ nanoparticles added to PANI, with a consequent reduction in conductivity as seen in figure 2. However, the small reduction in conductivity with increase in content of $\mathrm{ZrO}_{2}$ in PANI need not be considered as the negative result. As mentioned earlier, $\mathrm{ZrO}_{2}$ has the wide range of applications, which are all well studied. Therefore, we have tested its composites with PANI towards suitable material for supercapacitors.

\subsection{Electrochemical properties}

Electrochemical studies of pure $\mathrm{ZrO}_{2}$, PANI, PANI/ZrO ${ }_{2}^{-}$ 15 wt. $\%$ and PANI/ $\mathrm{ZrO}_{2}-25$ wt.\% electrodes were carried out by cyclic voltammetry (CV), chronopotentiometry (CP) and $\mathrm{AC}$ impedance techniques as describe below.

$\mathrm{CV}$ studies for the materials were carried out in the potential window -0.2 to $0.8 \mathrm{~V}$ in $1 \mathrm{M}$ aqueous $\mathrm{Na}_{2} \mathrm{SO}_{4}$ solution. Initially, the electrodes were stabilized for 20 cycles at the scan rate of $5 \mathrm{~m} \mathrm{Vs}^{-1}$ and $\mathrm{CV}$ experiments were carried out in different scan rates such as 2, 5, 10, 20, 40, 50, 80 and $100 \mathrm{~m} \mathrm{Vs}^{-1}$ to study the fast charge-discharge response. As perfect rectangular shaped $\mathrm{CV}$ patterns were not obtained (figure $5 \mathrm{a}$ ), the presence of small redox peaks around 0.4 and $0.5 \mathrm{~V}$ signifies that the nanocomposites exhibit pseudocapacitance behaviour [45]. The specific capacitance $\left(C_{\mathrm{sp}}\right)$ of 
the electrode materials were calculated using the following equation (6).

$$
C_{\mathrm{sp}}=\frac{1}{\gamma W(\Delta V)} \int_{V_{\mathrm{D}}}^{V_{\mathrm{c}}} i V \mathrm{~d} V,
$$

where $\gamma$ is the scan rate $\left(\mathrm{Vs}^{-1}\right), \mathrm{W}$ is active mass of material $(\mathrm{g})$ and $\Delta V$ is potential window $(\mathrm{V})$. Pure $\mathrm{ZrO}_{2}$, PANI and $\mathrm{PANI} / \mathrm{ZrO}_{2}-15 \mathrm{wt} . \%$ nanocomposites exhibited specific capacitance of 9,155 and $210 \mathrm{~F} \mathrm{~g}^{-1}$, respectively, whereas PANI $/ \mathrm{ZrO}_{2}-25$ wt.\% exhibited specific capacitance of 291 $\mathrm{F} \mathrm{g}^{-1}$ at $5 \mathrm{~m} \mathrm{Vs}^{-1}$. However, recently, Wei Zhang et al [36] have studied ultrafine nano $\mathrm{ZrO}_{2}$ as electrode for supercapacitors and found specific capacitance of $95 \mathrm{~F} \mathrm{~g} \mathrm{~g}^{-1}$ at scan rate of $5 \mathrm{~m} \mathrm{Vs}^{-1}$ by CV. These composite materials were also found to be stable even at lower scan rate of $2 \mathrm{~m} \mathrm{Vs}^{-1}$, indicating the electrode stability and exhibited higher specific capacitance values.

Figure $5 \mathrm{~b}$ shows the variation of specific capacitance with scan rate for the materials. It was also observed that the specific capacitance values were high for the nanocomposites at low scan rate indicating that there is easy movement of electrons from the electrode surface to the current collector during the reaction. The specific capacitance values decreases with increase in scan rate which is attributed to
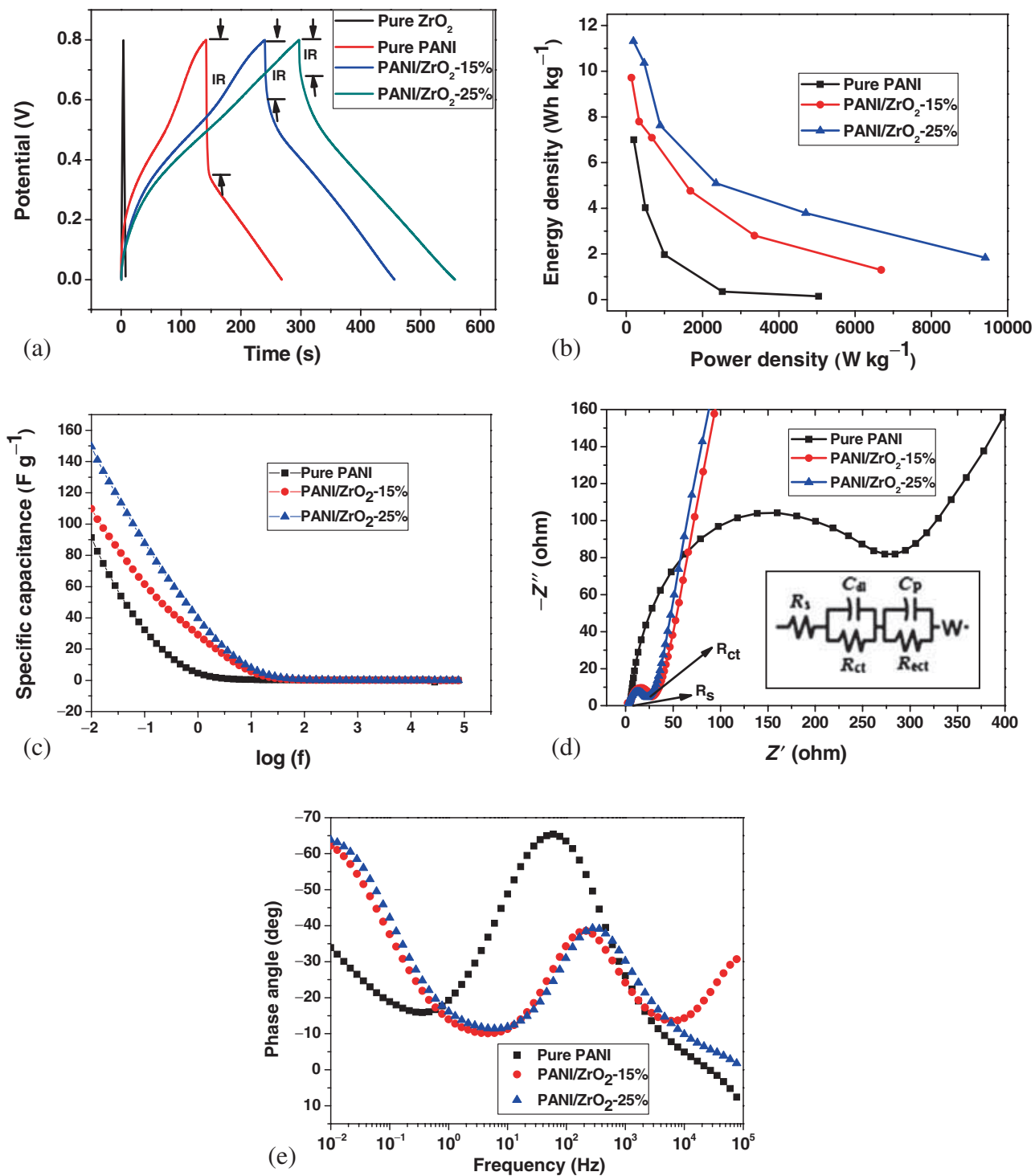

Figure 6. (a) GCD curves of pure $\mathrm{ZrO}_{2}$, PANI and PANI/ZrO $\mathrm{ZO}_{2}$ nanocomposites at a constant current of $0.2 \mathrm{~A} \mathrm{~g}^{-1}$. (b) Ragone plots related to energy density and power densities of pure PANI and $\mathrm{PANI} / \mathrm{ZrO}_{2}$ nanocomposites. (c) Specific capacitance values of pure PANI and $\mathrm{PANI} / \mathrm{ZrO}_{2}$ nanocomposites obtained from impedance data. (d) Nyquist plot of pure PANI and PANI/ZrO 2 nanocomposites with a frequency range from 0.01 to $10^{5} \mathrm{~Hz}$. Inset: equivalent circuit. (e) Bode plots of pure PANI and $\mathrm{PANI} / \mathrm{ZrO}_{2}$ nanocomposites. 
Table 2. Specific capacitance of pure $\mathrm{ZrO}_{2}$, PANI and $\mathrm{PANI} / \mathrm{ZrO}_{2}$ nanocomposites using chronopotentiometry.

\begin{tabular}{|c|c|c|c|c|}
\hline \multirow{2}{*}{$\begin{array}{l}\text { Current } \\
\text { density } \\
\left(\mathrm{A} \mathrm{g}^{-1}\right)\end{array}$} & \multicolumn{4}{|c|}{ Specific capacitance $\left(\mathrm{F} \mathrm{g}^{-1}\right)$} \\
\hline & $\begin{array}{l}\text { Pure } \\
\mathrm{ZrO}_{2}\end{array}$ & $\begin{array}{l}\text { Pure } \\
\text { PANI }\end{array}$ & $\mathrm{PANI} / \mathrm{ZrO}_{2}-15 \%$ & $\mathrm{PANI} / \mathrm{ZrO}_{2}-25 \%$ \\
\hline 0.2 & 11 & 79 & 109 & 127 \\
\hline 0.5 & 4 & 45 & 88 & 117 \\
\hline 1 & - & 22 & 80 & 86 \\
\hline 2.5 & - & 4 & 54 & 57 \\
\hline 5 & - & 2 & 32 & 43 \\
\hline 10 & - & - & 15 & 21 \\
\hline
\end{tabular}

incomplete utilization of the electroactive material at higher scan rates [45].

The charge-discharge behaviours of the materials were studied at the potential range $0-0.8 \mathrm{~V}$ at different applied current densities such as $0.2,0.5,1,2.5,5$ and $10 \mathrm{~A} \mathrm{~g}^{-1}$. Figure 6a shows CP curves of all electrode materials at the current density of $0.2 \mathrm{~A} \mathrm{~g}^{-1}$. The obtained $\mathrm{CP}$ curves were symmetry indicating good capacitive behaviour [46] of the composite materials. The $C_{\mathrm{sp}}$ was calculated by $\mathrm{CP}$ for the materials by using equation (7) and the values are tabulated in table 2.

$$
C_{\mathrm{sp}}=\frac{i}{W(\Delta V / \Delta t)},
$$

where $i$ is the current (A), ( $\Delta t)$ the discharge time (s), $(\Delta V)$ the potential window $(\mathrm{V})$ and $W$ the active mass of the electroactive material $(\mathrm{g})$. It was interesting to note that pure $\mathrm{ZrO}_{2}$ and PANI exhibited poor electrode performance and the $C_{\mathrm{sp}}$ are only 11 and $79 \mathrm{~F} \mathrm{~g}^{-1}$, respectively, at a current density of $0.2 \mathrm{~A} \mathrm{~g}^{-1}$. For PANI/ZrO $\mathrm{Zr}_{2}-15$ wt.\% and PANI/ $\mathrm{ZrO}_{2}-25$ wt.\% nanocomposite electrodes, the $C_{\mathrm{sp}}$ reach 109 and $127 \mathrm{~F} \mathrm{~g}^{-1}$ (table 2), respectively. The enhanced $C_{\text {sp }}$ may be due to the stabilized interaction between $\mathrm{ZrO}_{2}$ and PANI. Further, the voltage (IR) drop during discharge cycles are shown in figure $6 \mathrm{a}$ and were found to be $0.45,0.2$ and $0.12 \mathrm{~V}$ for pure PANI, PANI/ZrO $2-15$ wt. $\%$ and PANI/ $\mathrm{ZrO}_{2}-25$ wt.\% nanocomposite, respectively. The IR drop of $\mathrm{PANI} / \mathrm{ZrO}_{2}-25 \mathrm{wt} . \%$ is much lower than that of other two materials. The addition of $\mathrm{ZrO}_{2}$ nanoparticles to the PANI matrix has improved the specific capacitance due to the pseudocapacitance behaviour of $\mathrm{ZrO}_{2}$ nanoparticles. As reported by Chapal Kumar Das and group [47], the embedded $\mathrm{ZrO}_{2}$ in PANI matrix gives high energy storage capacity as it prevents aggregation and collapsing of PANI nanofibre. Further, highly conductive PANI assists in transportation of electron and ion diffusion into the matrix of $\mathrm{ZrO}_{2}$. Thus, there is a synergistic effect between the two different complementing properties of the materials, thereby enhancing the electrochemical performance of the nanocomposite with respect to IR drop. Cycle stability of the materials was studied at a current density of $0.5 \mathrm{~A} \mathrm{~g}^{-1}$ for 500 cycles. The calculated capacitance retention from the life cycle test for
PANI $/ \mathrm{ZrO}_{2}-25$ wt. $\%$ and $\mathrm{PANI} / \mathrm{ZrO}_{2}-15$ wt. $\%$ were 88 and $66 \%$, respectively. This improved cycle stability is presumed for the higher percentage of $\mathrm{ZrO}_{2}$ involved in charge storage mechanism. The energy density and power density of the composites were calculated using equations (8 and 9). Figure $6 \mathrm{~b}$ shows the Ragone plot for the electrode materials, exhibiting higher energy density of $11.32 \mathrm{Wh} \mathrm{kg}^{-1}$ at power density of $188 \mathrm{~W} \mathrm{~kg}{ }^{-1}$ for $\mathrm{PANI} / \mathrm{ZrO}_{2}-25 \mathrm{wt} . \%$ in comparison with pure PANI and $\mathrm{PANI} / \mathrm{ZrO}_{2}-15$ wt. $\%$.

$$
\begin{aligned}
& E=\frac{1}{2} C V^{2}, \\
& P=\frac{E}{t},
\end{aligned}
$$

where $E$ is the energy density ( $\mathrm{Wh} \mathrm{kg}^{-1}$ ), $C$ the specific capacitance $\left(\mathrm{F} \mathrm{g}^{-1}\right)$ using $\mathrm{CP}, V$ the potential window $(\mathrm{V}), P$ the power density $\left(\mathrm{W} \mathrm{kg}^{-1}\right)$ and $t$ the discharge time (s).

$\mathrm{AC}$ impedance studies were carried out for the electrode materials at $0.4 \mathrm{~V}$ in the frequency range of $0.01-10^{5} \mathrm{~Hz}$. The $C_{\mathrm{sp}}$ was estimated using equation (10) as in figure 6c using this technique was found to be 91,110 and $149 \mathrm{~F} \mathrm{~g}^{-1}$ for pure PANI, PANI/ZrO $\mathrm{Zr}_{2}-15$ wt. $\%$ and $\mathrm{PANI} / \mathrm{ZrO}_{2}-25$ wt. $\%$ nanocomposite electrodes, respectively.

$$
C_{\mathrm{sp}}=\frac{1}{2 \pi f Z^{\prime \prime}},
$$

where $f$ is the frequency $(\mathrm{Hz})$ and $Z^{\prime \prime}$ the imaginary part of impedance.

The Nyquist plots of pseudocapacitor materials such as transition metal oxide and conducting polymers are observed as a semicircle in the high frequency region and nearly a straight line along the imaginary axis at low frequency region as reported in the literature [36]. Figure 6d represents Nyquist plots for pure PANI and nanocomposites. In the low frequency region, the nanocomposites show more ideal capacitor behaviour; on the other hand, pure PANI exhibited a deviation from this ideal capacitor behaviour as in figure $6 \mathrm{~d}$ [48]. In the figure, the beginning of the semicircle corresponding to intercept on $x$ axis represents resistance $\left(R_{\mathrm{S}}\right)$ of the electrolyte in contact with the current collector and electrode. The end point of the semicircle region represents $\left(R_{\mathrm{ct}}\right)$ which is charge transfer resistance due to electrode material. The PANI $/ \mathrm{ZrO}_{2}-25$ wt. $\%$ exhibited $R_{\mathrm{s}}$ and $R_{\mathrm{ct}}$ as 2.77 and $20 \Omega$, respectively, which are relatively lower than that of pure PANI and PANI/ZrO $2-15$ wt.\%. The low values of $R_{\mathrm{S}}$ and $R_{\mathrm{ct}}$ are attributed to the $\mathrm{ZrO}_{2}$ nanoparticles dispersed in PANI and facilitate the efficient access of electrolyte ions to the PANI surface. The experimental data were fitted using Zsimpwin 3.21 software and the equivalent circuit (inset of figure $6 \mathrm{~d}$ ) consisting of double layer capacitance $\left(C_{\mathrm{dl}}\right)$, pseudocapacitance $\left(C_{\mathrm{p}}\right)$, solution resistance $\left(R_{\mathrm{S}}\right)$, charge transfer resistance $\left(R_{\mathrm{ct}}\right)$ and electron transfer resistance $\left(R_{\mathrm{ect}}\right)$ is represented for PANI/ZrO ${ }_{2}-25$ wt. $\%$. 
Figure 6e shows the Bode plots of all the electrode materials. The phase angle of $\mathrm{PANI} / \mathrm{ZrO}_{2}-25$ wt. $\%$ electrode was about $-64^{\circ}$ at a frequency of $0.01 \mathrm{~Hz}$, exhibiting nearly pure capacitive behaviour. Based on the frequency $(82.33 \mathrm{mHz})$ at a phase angle of $-45^{\circ}$ in which the capacitive and resistive impedances are equal, the calculated relaxation time for the same material was found to be $12 \mathrm{~s}$ [49]. The enhancement of specific capacitance by $\mathrm{CV}, \mathrm{CP}$ and $\mathrm{AC}$ impedance in the nanocomposites with increase in $\mathrm{ZrO}_{2}$ content is attributed to the electroactive $\mathrm{ZrO}_{2}$ nanoparticles present in PANI matrix as well as modified structure and morphology of the nanocomposite materials. $\mathrm{ZrO}_{2}$ behaves as pseudocapacitor material undergoing redox reaction during electrochemical process. The oxidation state of $\mathrm{Zr}$ in $\mathrm{ZrO}_{2}$ is +4 (higher oxidation state) is reduced to lower oxidation states (may be +3 or +2 ) in the presence of electrolyte giving out electrons which are responsible for its pseudocapacitor behaviour. PANI in the composite acts as conducting backbone for the electrons released during the redox reaction. These results imply that the nanocomposite materials can be good pseudocapacitors.

\section{Conclusion}

In summary, conducting $\mathrm{PANI} / \mathrm{ZrO}_{2}$ nanocomposites with varied percentage of $\mathrm{ZrO}_{2}$ were synthesized by chemical oxidative interfacial polymerization method. These nanocomposite materials were characterized and checked for their $\mathrm{AC}$ conductivity and electrochemical supercapacitance performance. AC conductivity of the nanocomposites obeys power law. The variation of AC conductivity and dielectric properties of the nanocomposites with varying $\mathrm{ZrO}_{2}$ concentration is attributed to the conformational changes in PANI matrix. The composites exhibit high dielectric constant and nearly a Debye type single relaxation. The PANI/ZrO nanocomposite prepared with $25 \mathrm{wt} . \%$ of $\mathrm{ZrO}_{2}$ nanoparticles in PANI showed higher capacitance of $341 \mathrm{~F} \mathrm{~g}^{-1}$ at a scan rate of $2 \mathrm{~m} \mathrm{Vs}^{-1}$ than that of other nanocomposite electrodes. In chronopotentiometric studies, this electrode was stable up to 500 cycles at a current density $0.5 \mathrm{~A} \mathrm{~g}^{-1}$ with $88 \%$ capacitance retention. It is also concluded that the composite with higher wt. \% of $\mathrm{ZrO}_{2}$ in the matrix of PANI is a better supercapacitor material which can be attributed to the maximized synergetic effect between PANI and $\mathrm{ZrO}_{2}$ nanoparticles.

\section{Acknowledgements}

We thank NRB - Naval Research Board-for the financial support given for this research work (project number: NRB290/MAT/12-13). We thank Dr Krishna Venkatesh, CEO, Centre for Emerging Technologies, Dr Chenraj Roychand, President, Jain University Trust, Dr M Krishna, Director, CMRTU and Prof B S Satyanarayana, Principal, RVCE, for their constant support in encouraging this research work.

\section{References}

[1] Wang Y Z, Gebler D D, Fu D K, Swager T M and Epstein A J 1997 Appl. Phys. Lett. 703215

[2] Yao B, Wang G, Ye J and Li X 2008 Mater. Lett. 621775

[3] Lina Geng, Yingqiang Zhao, Xueliang Huang, Shurong, Shoumin Zhang and Shihua Wu 2007 Sensor. Actuat. B Chem. 120568

[4] Khuspe G D, Bandgar D K, Sen Shashwati and Patil V B 2012 Synth. Met. 1621822

[5] Li D, Huang J and Kaner R B 2009 Acc. Chem. Res. 42135

[6] Li Q H, Wu J H, Tang Q W, Lan Z, Li P J, Lin J M and Fan L Q 2008 Electrochem. Commun. 101299

[7] Hui Guan, Li-Zhen Fan, Hongchang Zhang and Xuanhui Qu 2010 Electrochim. Acta 56964

[8] Jianhua L, Junwei A, Yecheng Z, Yuxiao M, Mengliu L, Mei Y and Songmei L 2012 ACS Appl. Mater. Interfaces 42870

[9] Snook G A, Kao P and Best A S 2011 J. Power Sources 1961

[10] Zhao L, Zhao L, Xu Y, Qiu T, Zhi L and Shi G 2009 Electrochim. Acta 55491

[11] Liu J L, Zhou M Q, Fan L Z, Li P and Qu X H 2010 Electrochim. Acta 555819

[12] Mandic Z, Rokovic M K and Pokupcic T 2009 Electrochim. Acta 542941

[13] Li J B, Jia Q M, Zhu J W and Zheng M S 2008 Polym. Int. 57337

[14] Tran H D, Wang Y, D‘Arcy J M and Kaner R B 2008 ACS Nano 21841

[15] Huang J X and Kaner R B 2004 J. Am. Chem. Soc. 126851

[16] Huang J X and Kaner R B 2004 Angew. Chem. Int. Ed. 43 5817

[17] Subramania A and Devi S L 2008 Polym. Adv. Technol. 19725

[18] Ghenaatian H R, Mousavi M F, Kazemi S H and Shamsipur M 2009 Synth. Met. 1591717

[19] Yuhong Jin, Shuo Huang, Mei Zhang and Mengqiu Jia 2013 Synth. Met. 16858

[20] Ahmad Abdolahi, Esah Hamzah, Zaharah Ibrahim and Shahrir Hashim 2012 Materials 51487

[21] Jiahua Zhu, Minjiao Chen, Honglin Qu, Xi Zhang, Huige Wei, Zhiping Luo et al 2012 Polymer 535953

[22] Yuhong Jin and Mengqiu Jia 2014 Synth. Met. 18947

[23] Wanga J-G, Yangb Y, Huanga Z-H and Kanga F $2012 \mathrm{~J}$. Power Sources 204236

[24] Chandramika Bora, Amarjyoti Kalita, Dhaneswar Das, Swapan K Doluia and Pratip Kr Mukhopadhyay 2013 Polym. Int. 63445

[25] Akash Katoch, Markus Burkhart, Taejin Hwang and Sang Sub Kim 2012 Chem. Eng. J. 192262

[26] Khuspe G D, Navale S T, Chougule M A, Shashwati Sen, Agawane G L, Kim J H and Patil V B 2013 Synth. Met. 1781

[27] Xiazhang Li, Chaoying Ni, Chao Yao and Zhigang Chen 2012 Appl. Catal. B Environ. 117-118 118

[28] Vassen R, Cao X, Tietz F, Basu D and Stover D 2000 J. Am. Cer. Soc. $\mathbf{8 3} 2023$

[29] Steele B C H and Heinzel A 2001 Nature 414345 
[30] Mohammad Bagher Gholivand and Leila Mohammadi-Behzad 2014 J. Electroanal. Chem. 71233

[31] Gupta K, Jana P C and Meikap A K 2011 J. Appl. Phys. 109 123713

[32] Rajeev Jain, Dinesh C Tiwari and Swati Shrivastava 2014 J. Electrochem. Soc. 161 B39

[33] Asif Ali Khan and Leena Paquiza 2011 Desalination 265242

[34] Tarique Anwer, Mohd Omaish Ansari and Faiz Mohammad 2013 J. Ind. Eng. Chem. 191653

[35] Nasibi M, Golozar M A and Rashed G 2012 J. Power Sources 206108

[36] Wei Zhang, Yueyue Tan, Yilong Gao, Jianxiang Wu and Bohejin Tang 2015 Ceram. Int. 412626

[37] Ko Y N, Choi S H, Kang Y C and Park S B 2013 ACS Appl. Mater. Interf. 53234

[38] Yinhai Zhu, Enhui Liu, Zhenyu Luo, Tiantian Hu, Tiantian Liu, Zengpeng Li and Qingling Zhao 2014 Electrochim. Acta 118106

[39] Sahu H R and Rao G R 2000 B. Mater. Sci. 23349
[40] Bowen C R, Dent A C E, Almond D P and Comyn T P 2008 Ferroelectrics $\mathbf{3 7 0} 166$

[41] Jing Jiang, Lun-Hong Ai, Da-Bin Qin, Hui Liu and Liang-Chao Li 2009 Synth. Met. 159695

[42] Kim H M, Lee C Y and Joo J 2000 J. Kor. Phys. Soc. 36371

[43] Hazarika J and Kumar A 2014 Synth. Met. 198239

[44] Hui D, Alexandrescu R, Chipara M, Morjan I, Aldica Gh, Chipara M D and Lau K T 2004 J. Optoelect. Adv. Mater. 6817

[45] Farrukh Iqbal Dar, Kevin Radakishna Moonooswamy and Mohammed Es-Souni 2013 Nanoscale Res. Lett. 81

[46] Zhe-Fei Li, Hangyu Zhang, Qi Liu, Yadong Liu, Lia Stanciu and Jian Xie 2014 Carbon 7I 257

[47] Soumen Giri, Debasis Ghosh and Chapal Kumar Das 2014 Adv. Funct. Mater. 241312

[48] Zan Gao, Wanlu Yang, Jun Wang, Huijun Yan, Yuan Yao, Jing Ma et al 2015 Electrochim. Acta 91185

[49] Xu Ji, Shuang Cheng, LufengYang, Yu Jiang, Zhong-Jie Jiang, Chenghao Yang et al 2015 Nano Energy 11736 